\title{
Lorentz Force from a Current-Carrying Wire on a Charge in Motion under the Assumption of Neutrality in the Symmetrical Frame of Reference
}

\author{
Antonio Šunjerga1,2, Franjo Sokolić², Marcos Rubinstein ${ }^{3}$, Farhad Rachidi1 \\ ${ }^{1}$ Electromagnetic Compatibility Laboratory, EPFL, Switzerland \\ ${ }^{2}$ Department of Physics, Faculty of Science, Split, Croatia \\ ${ }^{3}$ University of Applied Sciences Western Switzerland, Yverdon-les-Bains, Switzerland \\ Email: antonio.sunjerga@epfl.ch
}

How to cite this paper: Šunjerga, A., Sokolić, F., Rubinstein, M. and Rachidi, F. (2018) Lorentz Force from a Current-Carrying Wire on a Charge in Motion under the Assumption of Neutrality in the Symmetrical Frame of Reference. Journal of Modern Physics, 9, 2473-2481.

https://doi.org/10.4236/jmp.2018.914159

Received: February 21, 2018

Accepted: December 23, 2018

Published: December 26, 2018

Copyright (c) 2018 by authors and Scientific Research Publishing Inc. This work is licensed under the Creative Commons Attribution International License (CC BY 4.0).

http://creativecommons.org/licenses/by/4.0/

\begin{abstract}
It is commonly assumed that a wire conducting an electric current is neutral in the laboratory frame of reference (the rest frame of the lattice of positive ions). Some authors consider that the wire is neutral only in a symmetrical frame of reference, in which the velocities of electrons and protons have equal norm and opposite direction. In this paper, we discuss the Lorentz transformation between different frames of reference in the context of the special theory of relativity for a current-carrying conducting wire and a probe charge in motion with respect to the wire. A simple derivation of the Lorentz force in the laboratory frame of reference for the assumed neutrality in a symmetrical frame of reference is presented. We show that the Lorentz force calculated assuming neutrality in the symmetrical frame of reference and the one assuming neutrality in the laboratory frame of reference differ by a term corresponding to a change in the test charge speed of one half the drift velocity of the electrons.
\end{abstract}

\section{Keywords}

Special Theory of Relativity, Current-Carrying Wire, Neutral Frame, Symmetrical Frame, Lorentz Force, Drift Velocity

\section{Introduction}

The Lorentz force between a current-carrying wire and a charge in motion in the laboratory frame of reference, where the conductor is at rest, is often expressed as: 


$$
\boldsymbol{F}=q \boldsymbol{v} \times \boldsymbol{B}
$$

where $\boldsymbol{B}$ is the magnetic flux density generated by the wire current, which can be calculated by using Biot-Savart's Law [1]. $\boldsymbol{B}$ depends only on the current magnitude regardless of the physics of the motion of the charge carriers in the wire. In (1), $q$ is the charge and $\boldsymbol{v}$ is its velocity. Equation (1) is correct only under the assumption that the wire is neutral in the laboratory reference frame. Otherwise, a second component of the force due to the electric field should also be considered.

The question of the frame of reference, in which the current-carrying wire is neutral, has been the subject of debate in the past years [2]-[10]. Some authors suggest that a neutral wire corresponds to the rest reference frame of the lattice of positive ions (e.g., [2] [6] [7] [8]), considering that electrons are a free ensemble and, therefore, their distances do not change upon acceleration [3]. Others (e.g., [4] [9]) assume that the distances between electrons are also subject to the Lorentz contraction and, therefore, the wire is neutral only in a symmetrical frame of reference in which both electrons and protons have the same speed but move in opposite directions. Although they are conceptually important, to the best of our knowledge none of these theories has been experimentally proven since the drift velocity of the electrons is small and, hence, the effects are negligible. It is therefore necessary to investigate the mechanisms involved in the transition process from no-current wire to current-carrying wire to answer the question of the neutral frame. A more detailed discussion about the issue of the determination of the reference frame in which the current-carrying wire is neutral can be found in [2] and [3].

In this paper, we will first present a simple derivation for the Lorentz force by assuming the Lorentz contraction of distances between electrons and assuming that the wire neutrality occurs in a symmetrical frame of reference (as in [4] [9]). This assumption leads to a modification of (1). The Lorentz force will depend on the physics of the motion of the charges. In Section 2, we will derive the Lorentz force for the classical example of a charge moving parallel to a current-carrying wire [10] (see Figure 1 for the geometry of the problem), for a symmetrical frame of reference. In Section 3, we will derive the same force considering a laboratory frame of reference (at rest with respect to the lattice of positive ions), by transforming the force from the symmetrical frame of reference. Conclusions will be given in Section 4 .

\section{Symmetrical Frame of Reference}

Let us first examine the theoretical model from [10]. Figure 2 shows the problem considering two different reference frames moving with respect to each other at a speed $v$. The first one (left panel) will be labeled $S$, and the second (right panel) $S^{\prime}$.

Let us imagine that in the symmetrical frame of reference $S$, we have a straight, infinitely-long wire containing positive and negative charges, characterized by 


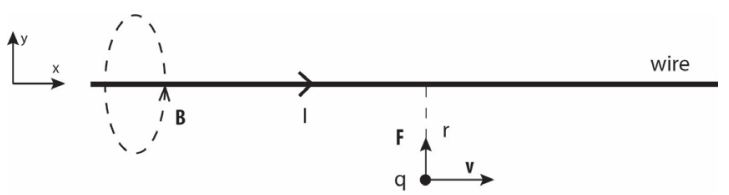

Figure 1. Force between a current-carrying wire and a charge $q$ at a distance $r$. The charge is moving in the direction parallel to the wire with a speed $v$.

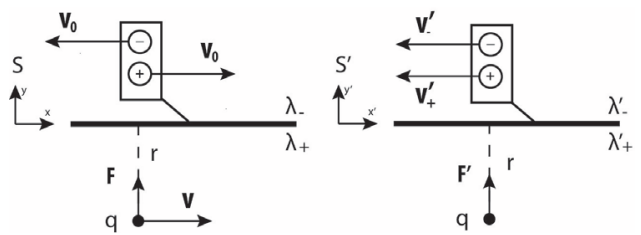

Figure 2. Frame of reference $S$ (left panel) and frame of reference $S^{\prime}$ (right panel) for $|\nu|>$ $\left|v_{0}\right|$. Probe charge $q . \lambda_{+}$and $\lambda_{-}$are the line charge densities in S. $\lambda_{+}^{\prime}$ and $\lambda_{-}^{\prime}$ are the line charge densities in $S^{\prime}$.

linear charge densities $\lambda_{+}$and $\lambda_{-}$. The positive wire charges move with a constant velocity $v_{0}$ in the positive $x$ direction, while the negative wire charges move with a constant velocity- $V_{0}$. These two charge densities are measured in the $S$ frame of reference (Figure 2, left panel), with respect to which the positive and negative charges are moving. If we assume that both charge densities are equal in absolute value $\left(\left|\lambda_{+}\right|=\left|\lambda_{-}\right|\right)$, the wire is electrically neutral. Now, let us examine a probe charge $q$ at a distance $r$ moving with a velocity $\mathrm{v}$ along a line parallel to the wire in S. In what follows, we will refer to $q$ as the probe charge and to the charges composing the linear charge densities as wire charges.

Let us calculate the force on the probe charge $q$. Frame $S^{\prime}$ is moving with a speed $v$ relative to $S$ so that the probe charge $\mathrm{q}$ is not in motion in $S^{\prime}$. The velocities of the positive and negative line charges in $\mathrm{S}^{\prime}$ can be calculated from the velocities in $\mathrm{S}$ by way of the Lorentz transformation:

$$
v_{+}^{\prime}=\frac{v_{0}-v}{1-\frac{v v_{0}}{c^{2}}}, v_{-}^{\prime}=\frac{\left(-v_{0}\right)-v}{1-\frac{v\left(-v_{0}\right)}{c^{2}}}
$$

Clearly, if $v$ is not equal to zero, $v_{+}^{\prime}$ and $v_{-}^{\prime}$ will have different values. Consequently, the distance between the individual positive charges will experience a different contraction compared to the distance between the individual negative charges. We therefore expect different line charge densities $\lambda_{+}^{\prime}$ and $\lambda_{-}^{\prime}$ in $S^{\prime}$. As a result, the wire will not be neutral in S'.

Let us introduce the following parameters [10]:

$$
\begin{gathered}
\beta=\frac{v}{c}, \gamma=\frac{1}{\sqrt{1-\beta^{2}}} \\
\beta_{0}=\frac{v_{0}}{c}, \gamma_{0}=\frac{1}{\sqrt{1-\beta_{0}^{2}}} \\
\beta_{ \pm}^{\prime}=\frac{v_{ \pm}^{\prime}}{c}, \gamma_{ \pm}^{\prime}=\frac{1}{\sqrt{1-\beta_{ \pm}^{\prime 2}}}
\end{gathered}
$$


By dividing (2) by $\mathcal{C}$, we can now rewrite $\beta_{+}^{\prime}$ and $\beta_{-}^{\prime}$ as [10]:

$$
\beta_{+}^{\prime}=\frac{\beta_{0}-\beta}{1-\beta_{0} \beta}, \beta_{-}^{\prime}=-\frac{\beta_{0}+\beta}{1+\beta_{0} \beta}
$$

Let us now consider two other frames of reference: 1) the rest frame of reference of the positive wire charges $S_{+}$moving with respect to $S$ with velocity $v_{0}$ in the positive $x$ axis direction, and, similarly, 2) the rest frame of reference of the negative wire charges, $S_{-}$moving with respect to $S$ with a velocity $-V_{0}$. Since the linear charge densities are the same in $S$ and since their rest reference frames, $S_{+}$ and $S_{-}$, are moving with the same speed with respect to $S$, they experience the same contraction. As a result, the linear charge density of the negative charges in

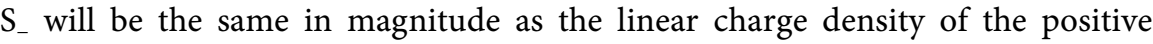
charges in $\mathrm{S}_{+}$. If we label the magnitude of the linear charge density in $\mathrm{S}$ as $\lambda(\lambda=$ $\left.\left|\lambda_{+}\right|=\left|\lambda_{-}\right|\right)$, the linear charge density in the rest frame of reference is simply $\pm \lambda / \gamma_{0}$, where the sign depends on the wire charge polarity. Now, transforming from $S$ to $S$, the linear charge density in terms of $\lambda$ can be expressed as:

$$
\lambda_{+}^{\prime}=\gamma_{+}^{\prime} \frac{\lambda}{\gamma_{0}}, \lambda_{-}^{\prime}=-\gamma_{-}^{\prime} \frac{\lambda}{\gamma_{0}}
$$

From Equation (7), the overall charge density in $S^{\prime}$ can be expressed as [10]:

$$
\begin{aligned}
\lambda_{\text {overall }}^{\prime} & =\lambda_{+}^{\prime}+\lambda_{-}^{\prime}=\gamma_{+}^{\prime} \frac{\lambda}{\gamma_{0}}-\gamma_{-}^{\prime} \frac{\lambda}{\gamma_{0}}=\frac{\lambda}{\gamma_{0}}\left(\frac{1}{\sqrt{1-\frac{v_{+}^{\prime 2}}{c^{2}}}}-\frac{1}{\sqrt{1-\frac{v_{-}^{\prime 2}}{c^{2}}}}\right) \\
& =\frac{\lambda}{\gamma_{0}}\left(\frac{1}{\sqrt{1-\beta_{+}^{\prime 2}}}-\frac{1}{\sqrt{1-\beta_{-}^{\prime 2}}}\right)=-2 \lambda \beta \beta_{0} \gamma=-\frac{2 \lambda \gamma v v_{0}}{c^{2}}
\end{aligned}
$$

The electric field from a uniform line charge in $S^{\prime}$ at the position of the probe charge is given by:

$$
E_{y}^{\prime}(r)=-\frac{\lambda_{\text {overall }}^{\prime}}{2 \pi \epsilon_{0} r}
$$

As mentioned before, no magnetic force is exerted on the charge since its speed is zero. The total force can therefore be calculated as

$$
F_{y}^{\prime}(r)=-\frac{q \lambda_{\text {overall }}^{\prime}}{2 \pi \epsilon_{0} r}
$$

Making use of the Lorentz transformation, the force in reference frame $\mathrm{S}$ is [10]:

$$
F_{y}(r)=\frac{F_{y}^{\prime}}{\gamma}=\frac{q \lambda v v_{0}}{\pi \epsilon_{0} c^{2} r}
$$

Now, considering that

$$
I=\lambda v_{0}+(-\lambda)\left(-v_{0}\right)=2 \lambda v_{0} \text { and } B(r)=\frac{I}{2 \pi \epsilon_{0} c^{2} r}
$$

and plugging these relations into (11), we obtain the more familiar equation: 


$$
F_{y}(r)=q v B
$$

This classical example is often used to show the relativistic background of equation (1). In the following section, we will use this derivation to get an expression for the Lorentz Force in the laboratory frame of reference.

\section{Laboratory Frame of Reference (Rest Frame of Reference of a Lattice of Positive Charges)}

Let us now consider the same case of an infinitely-long current-carrying wire in a laboratory frame of reference. If there is no applied voltage, the random motion of the charges is described with quantum mechanics [11]. If we apply a voltage on the wire, the motion of the electrons will be a superposition of their random motion and that caused by the applied electric field. To a first approximation, the motion can be described as if all electrons were moving with a constant drift velocity [12]. Positive charges are stationary (in lattice) in the considered frame of reference, referred to as the laboratory frame of reference (shown in Figure 3). It is assumed that a voltage is applied between the wire ends at infinity, with the left end at a higher potential. The charge $q$ is at a distance $r$ to the wire and it moves with a constant velocity $v_{\text {qlab }}$ in the positive $\mathrm{x}$ direction, parallel to the wire.

We will now calculate the force applied on the charge in this frame of reference. First, we will transform to the symmetrical frame $S$ (in which the wire is assumed to be neutral) where we will call the speeds of the positive and of the negative charges $v_{+}$and $v_{-}$such that:

$$
v_{+}=-v_{-}=v_{o}
$$

In this frame of reference, the force is given by Equation (11), in which the value of $v$, which will be calculated below, is the velocity of the test charge ( $v_{\text {qlab }}$ in the laboratory frame of reference) with respect to the symmetrical frame of reference. The Lorentz transformation of the positive and negative wire charge velocities to $S$ from $S_{\text {lab }}$ is given by

$$
v_{+}=-v_{S}, \quad v_{-}=\frac{\left(-v_{D}\right)-v_{S}}{1-\frac{v_{S}\left(-v_{D}\right)}{c^{2}}}
$$

in which $v_{s}$ is the velocity of the frame of reference $S$ with respect to $S_{\text {lab. }}$.

Substituting Equation (15) in (14), we obtain:

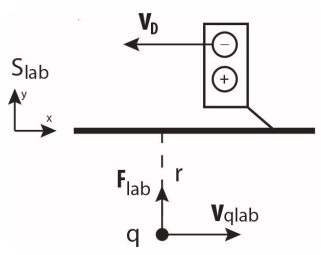

Figure 3. Laboratory frame of reference. Electrons are moving with the drift velocity $v_{\mathrm{D}}$ and positive ions are stationary. The rectangle above the wire represents a differential wire segment illustrating the speeds of the positive and negative charges. 


$$
\frac{v_{D}}{c^{2}} v_{S}^{2}-2 v_{S}-v_{D}=0
$$

Since the drift velocity $v_{D}$ is in the order of a few $\mathrm{mm} / \mathrm{s}$ and $v_{S}$ is even smaller, to a first approximation we can neglect the first term in Equation (16) and obtain the classical Galilean transformation:

$$
v_{S}=-\frac{v_{D}}{2}
$$

In the symmetrical reference frame $S$, the velocity of the charge is, therefore:

$$
v=\frac{v_{q l a b}-\left(-\frac{v_{D}}{2}\right)}{1-\frac{\left(-\frac{v_{D}}{2}\right) v_{q l a b}}{c^{2}}}
$$

Using Equation (11), the force in the reference frame $S$ becomes:

$$
F_{y}=\frac{q \lambda v \frac{v_{D}}{2}}{\pi \epsilon_{0} c^{2} r}
$$

In which $\lambda$ is the magnitude of the positive or the negative charge density in the symmetrical frame of reference in the same way as it was defined in the previous section $\left(\lambda=\left|\lambda_{+}\right|=|\lambda|\right)$. The Lorentz transformation of this force to the laboratory frame of reference $S_{\text {lab }}$ can be expressed as:

$$
F_{y l a b}=\frac{F_{y}}{\gamma_{S}\left(1-\frac{v\left(-\frac{v_{D}}{2}\right)}{c^{2}}\right)}=\frac{q \lambda v \frac{v_{D}}{2}}{\gamma_{S}\left(1+\frac{v \frac{v_{D}}{2}}{c^{2}}\right) \pi \epsilon_{0} c^{2} r}
$$

where:

$$
\gamma_{S}=\frac{1}{\sqrt{1-\frac{v_{s}^{2}}{c^{2}}}}
$$

The negative charge density in $\mathrm{S}_{\text {lab }}$ is:

$$
\lambda_{l a b-}=-\frac{\lambda}{\gamma_{S}} \gamma_{x}
$$

in which

$$
\gamma_{x}=\frac{1}{\sqrt{1-\left(\frac{v_{D}}{c}\right)^{2}}}
$$

Let us now define:

$$
\alpha=\frac{1}{1+\frac{\frac{v_{D}}{2} v_{q l a b}}{c^{2}}} \text { and } \delta=\frac{1}{1+\frac{v \frac{v_{D}}{c^{2}}}{c^{2}}}
$$


Plugging (18) into (20), expressing $\lambda$ in terms of $\lambda_{\text {lab- }}$ from (20), and using (23) and (24), the Lorentz force in $S_{\text {lab }}$ is:

$$
\begin{aligned}
F_{y l a b}= & \frac{q \gamma_{\mathrm{S}}\left(-\lambda_{\text {lab- }}\right)\left(\frac{v_{q l a b}+\frac{v_{D}}{2}}{\alpha}\right) \frac{v_{D}}{2}}{\gamma_{\mathrm{S}} \gamma_{x} \delta \pi \epsilon_{0} c^{2} r} \\
= & \frac{q\left(v_{q l a b}+\frac{v_{D}}{2}\right) I}{2 \gamma_{x} \delta \alpha \pi \epsilon_{0} c^{2} r}=\frac{q\left(v_{q l a b}+\frac{v_{D}}{2}\right) B}{\gamma_{x} \delta \alpha}
\end{aligned}
$$

where $B$ and $I$ are defined for the laboratory frame of reference, and where the current is only due to the motion of the negative charges.

Since $v_{D} \ll c$, the relativistic coefficient in Equations (23) and (24) is approximately equal to one and (25) can be written as:

$$
F_{y l a b} \approx q\left(v_{q l a b}+\frac{v_{D}}{2}\right) B
$$

In the laboratory frame of reference (rest frame of the lattice of positive charges), the negative wire charges are moving, as opposed to the positive charges that are stationary. Therefore, they have a higher linear charge density than the stationary positive charges. The overall wire is negatively charged and it produces an electric force in the direction of the magnetic force in the examined case. If the probe charge is moving in the positive direction of the $\mathrm{x}$ axis as in Figure 3 (opposite to the drift of the electrons), force in (1) is increased by $q v_{D} B / 2$ in (26).

It is worth noting that in opposite case when drift velocity of negative wire charges is positive, the sign of force due to probe charge movement will change due to change of sign of current and consequently of magnetic field. However, contribution of this half drift velocity correction term will remain to be directed toward the wire due to change of sign in (17). This is because wire will again be negatively charged in laboratory frame. There will be also change in denominator of two terms in (24), but these terms can be neglected as in (26).

Figure 4 shows comparison of forces calculated in the laboratory frame of reference assuming neutrality in $S_{\text {lab }}$ and in the symmetrical frame of reference $S$ for the case of $B=1 \mathrm{~T}$ and $q=1 \mathrm{C}$. As the charge velocity increases, the difference between the forces becomes negligible. The drift velocity [12] is very small, making these effects hard to measure since they are hidden by some other real-scenario forces, such as the zero-order effect of the electrostatic force created by an image charge inside the conducting wire, the first-order force resulting from the resistive nature of the wire, and second-order forces originating for example from the curvature of the wire [4] [13]. In order to mitigate the dominant effect of the zero-order effect force, one may exploit the fact that this force will decay as $1 / r^{2}$ while (26) exhibits a slower $1 / r$ decay. 


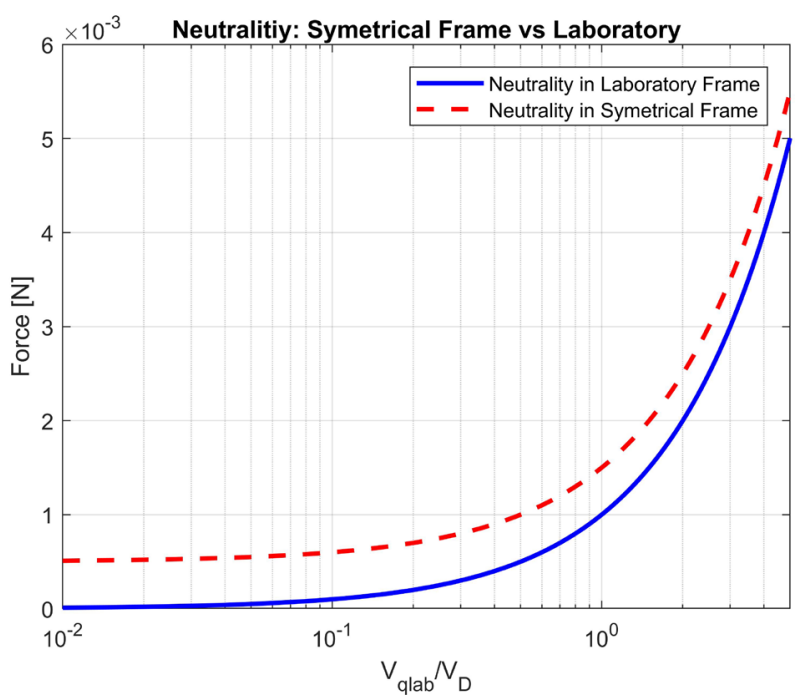

(b)

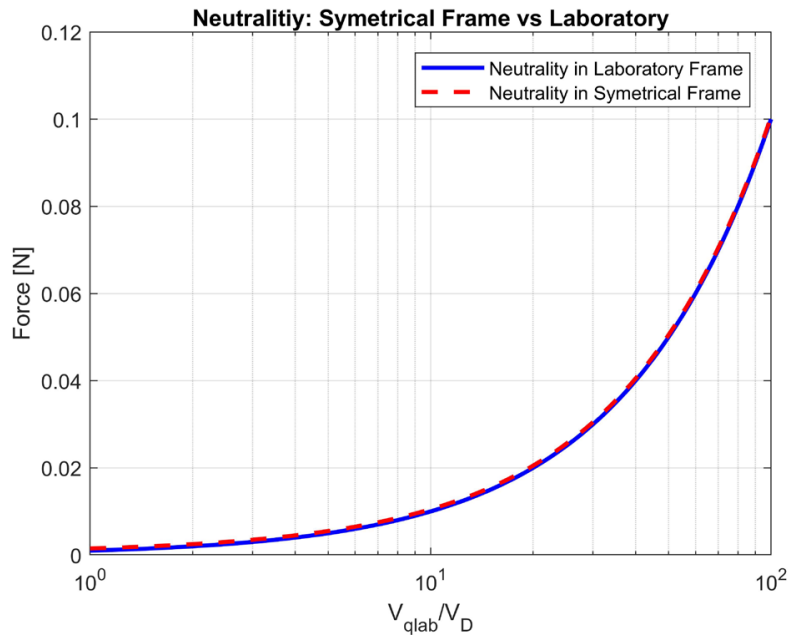

(b)

Figure 4. Force applied on the charge calculated assuming neutrality in the laboratory frame of reference given with Equation (26) (dashed red curve) and in the symmetrical S frame of reference given by Equation (1) (solid blue curve). For low values of the $V_{\text {qlab }} / V_{D}$ ratio (a) and for high values (b). For the case of $v_{D}<0$ and $v_{q l a b}>0$.

\section{Conclusion}

In this paper, we presented a derivation of the Lorentz force in the laboratory frame of reference for the case of a metallic, current-carrying wire under the assumption of neutrality in the symmetrical frame of reference. The Lorentz force is a combination of the electric and the magnetic forces and, depending on the physics of motion of the charges, the electric field will also be present. We showed that the Lorentz force calculated assuming neutrality in the symmetrical frame of reference and the one assuming neutrality in the laboratory frame of reference differ by a term corresponding to a change in the test charge speed of one half the drift velocity of the electrons. The derived equations make it in principle possible to experimentally test the hypotheses of neutrality. The drift 
velocity being usually in the order of $\mathrm{mm} / \mathrm{s}$, an accurate measurement of these effects might be, however, very challenging, compared to other forces that are in play.

\section{Acknowledgements}

Special thanks to Prof. Ante Bilušić for all the great and enthusiastic lectures. Sincere thanks to the two anonymous reviewers whose constructive comments and suggestions improved this paper.

\section{Conflicts of Interest}

The authors declare no conflicts of interest regarding the publication of this paper.

\section{References}

[1] Jackson, J. (1999) Classical Electrodynamics. 3rd Edition, John Wiley \& Sons, New York.

[2] Redzic, D.V. (2011) European Journal of Physics, 33, 513-523. https://doi.org/10.1088/0143-0807/33/3/513

[3] Folman, R. (2013) Journal of Physics. Conference Series, 437, 012013. https://doi.org/10.1088/1742-6596/437/1/012013

[4] Brill, M.H., Dameron, D. and Phipps Jr., T.E. (2011) Physics Essays, 24, 325-326. https://doi.org/10.4006/1.3593402

[5] Redzic, D.V. (2014) Serbian Astronomical Journal, No. 188, 55-65. https://doi.org/10.2298/saj1488055r

[6] Feynman, R.P., Leighton, R.B. and Sands, M.L. (2010) The Feynman Lectures on Physics. Volume II New Millenium Edition, New York.

[7] van Kampen, P. (2008) European Journal of Physics, 29, 879-883. https://doi.org/10.1088/0143-0807/29/5/002

[8] Gabuzda, D.C. (1993) American Journal of Physics, 61, 360-362. https://doi.org/10.1119/1.17271

[9] Peters, P.C. (1985) American Journal of Physics, 53, 1165-1169. https://doi.org/10.1119/1.14075

[10] Belsley, M. (2013) Contemporary Physics, 54, 261-262. https://doi.org/10.1080/00107514.2013.836250

[11] Kittell, C. (2005) Introduction to Solid State Physics. 8th Edition, John Wiley \& Sons, New York.

[12] Halliday, D., Resnick, R. and Walker, J. (2014) Fundamentals of Physics. 10th Edition, John Wiley \& Sons, Hoboken, NJ.

[13] Assis, A.K.T. and Hernandes, J.A. (2007) The Electric Force of a Current. C. Roy Keys, Montreal. 